\title{
CONSTRUCTION OF FFAG ACCELERATORS IN KURRI FOR ADS STUDY
}

\author{
M. Tanigaki*, K. Mishima, S. Shiroya, RRI, Kyoto University, Kumatori, Osaka 590-0494, Japan \\ Y. Ishi, S. Fukumoto, Mitsubishi Electric Company, Kobe, 652-8555, Japan \\ S. Machida, Y. Mori, KEK, Tsukuba, Ibaragi 305-0801, Japan \\ M. Inoue, SLLS, Ritsumeikan University, Kusatsu, Shiga 525-8577, Japan
}

\begin{abstract}
Kumatori Accelerator driven Reactor Test project (KART) has been started at Kyoto University Research Reactor Institute (KURRI) from the fiscal year of 2002, aiming to demonstrate the basic feasibility of ADS and to develop an $150 \mathrm{MeV}$ proton FFAG (Fixed Field Alternating Gradient) accelerator complex as a neutron production driver. This FFAG complex will be connected with our Kyoto University Critical Assembly (KUCA) for the basic ADS experiments by the end of March 2006.
\end{abstract}

\section{INTRODUCTION}

As a substitute for the $5 \mathrm{MW}$ reactor at Kyoto University (KUR), a neutron source based on the ADS concept has been proposed in 1996[1]. The conceptual design study on ADSR using the MCNPX code clarified the lack of reliable effective multiplication factor $k_{e f f}$ in the proton energy region between $20 \mathrm{MeV}$ and $150 \mathrm{MeV}$. Since the experimental studies in our institute were performed using KUCA and a $300 \mathrm{keV}$ Cockcroft-Walton accelerator[2, 3], a proton beam source which covers between $20 \mathrm{MeV}$ and $150 \mathrm{MeV}$ is required to extend our study on ADS system.

The requirements towards proton sources for ADS are 1) high beam intensity, 2) high efficiency on power consumption, and 3) high stability in operation. FFAG accelerator which was orignally proposed by Ohkawa 40 yeasrs ago[4] is regarded as a good candidate as the proton driver for ADS. Because of its fixed magnetic field the high repetition rate of beam acceleration and the much less power consumption in the accelerator by the introduction of superconducting magnet are expected. Although such attractive features, except electron models, no FFAG with RF has not been realized until recently because of technical difficulties such as the production of wide band high voltage RF cavity or the lack of a long straight section for beam injection and extraction. Recently, Mori et al. have developed a wide band RF cavity with FINEMET[5] and succeeded the first acceleration of proton with a $500 \mathrm{keV}$ PoP FFAG synchrotron[6]. Now they have developed a "returnyoke free" magnet for the $150 \mathrm{MeV}$ FFAG synchrotron[7] in which they try to extract the beam from FFAG for the first time.

On such basis of our study and the technical developments on FFAG, KART project has been approved and started from the fiscal year of 2002. In this project, the basic feasibility of ADS system and the multiplication factor $k_{\text {eff }}$ in the energy region of $\mathrm{E}_{p}=20 \sim 150 \mathrm{MeV}$ will be studied. Another important aim in this project is to develop a practical FFAG accelerator as a proton driver for ADS.

\section{FFAG ACCELERATOR COMPLEX}

In KART project, an FFAG accelerator complex is now under construction as the proton source for ADS study. This complex consists of one FFAG with an induction unit for acceleration as the injector and two FFAG with RF as the booster and main accelerators, respectively. All of these accelerators will be in pulse operation at the repetition rate of $100 \mathrm{~Hz}$. The schematic diagram of our FFAG complex is shown in Fig. 1. Basic specifications for this FFAG complex are summarized in Table 1. The layout of these FFAG accelerators in the accelerator room is shown in Fig. 2.

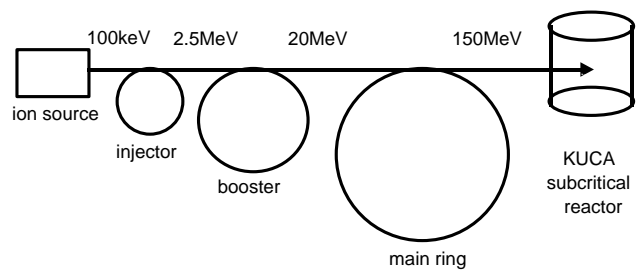

Figure 1: The schematic diagram of FFAG complex at KURRI.

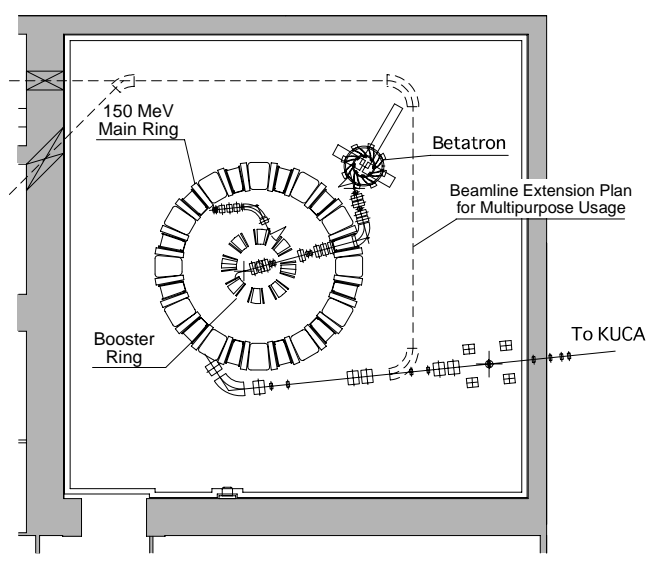

Figure 2: FFAG complex at KURRI.

\footnotetext{
*tanigaki@ @ri.kyoto-u.ac.jp
} 
Table 1: Specification of FFAG complex

\begin{tabular}{cccc}
\hline & Injector & Booster & Main \\
\hline Focusing & Spiral & Radial & Radial \\
Acceleration & Induction & Synchrotron & Synchrotron \\
$k$ & 2.5 & 4.5 & 7.6 \\
$\mathrm{E}_{i n j}$ & $100 \mathrm{keV}$ & $2.5 \mathrm{MeV}$ & $20 \mathrm{MeV}$ \\
$\mathrm{E}_{\text {ext }}$ & $2.5 \mathrm{MeV}$ & $20 \mathrm{MeV}$ & $150 \mathrm{MeV}$ \\
$p_{\text {ext }} / p_{i n j}$ & 5.00 & 2.84 & 2.83 \\
$r_{i n j}$ & $0.60 \mathrm{~m}$ & $1.42 \mathrm{~m}$ & $4.54 \mathrm{~m}$ \\
$r_{\text {ext }}$ & $0.99 \mathrm{~m}$ & $1.71 \mathrm{~m}$ & $5.12 \mathrm{~m}$ \\
\hline
\end{tabular}

\section{Ion source}

The ion source in this FFAG complex is a typical multicusp type. $\mathrm{H}^{+}$ions are extracted and accelerated to 100 $\mathrm{keV}$, then transported to the injector. Since all of the FFAG complex are operated in pulse mode, the beam is bunched by an electrostatic beam chopper in the transport line.

\section{Injector FFAG with an induction unit for accel- eration}

In the present FFAG complex, a 2.5 MeV FFAG with an induction unit for acceleration is used as the injector. This FFAG has 12 sprial sector magnets with the spiral angle of 42 degrees. A typical pattern of induced acceleration voltage is shown in Fig. 4. In this operation pattern, proton beam from the ion source is accepeted for $50 \mu \mathrm{s}$ and the beam pulse ejected from the injector is compressed to $5 \mu \mathrm{s}$.

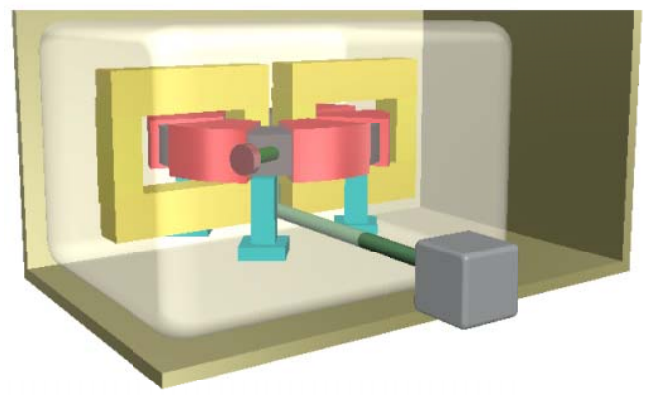

Figure 3: Schematic view of the FFAG injector.

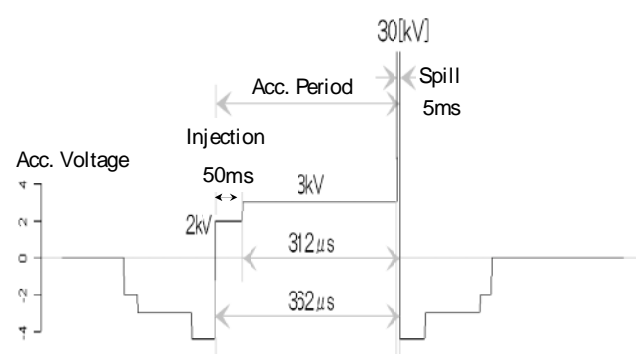

Figure 4: Acceleration pattern of FFAG injector.

\section{Booster FFAG Synchrotron}

Injected beam from the FFAG injector at the energy of $2.5 \mathrm{MeV}$ is then accelerated up to $20 \mathrm{MeV}$ in this booster ring. This FFAG synchrotron is the radial sector type, consisting of 8 cells of DFD magnets. The lattice structure of this booster ring is shown in Fig. 5. These magnets are "return-yoke free" magnets with flat pole face and each magnet has 22 trim coils placed along the $r$ direction to produce the magnetic field following the function of $B=B_{0}\left(r / r_{0}\right)^{k}$. The advantage of using trim coils is that one can easily change the $k$ by choosing proper current set to trim coils, which is an important characteristics for the variable energy FFAG accelerators. An 2D simulation of FFAG magnetic field calculated by POISSON is shown in Fig. 6.

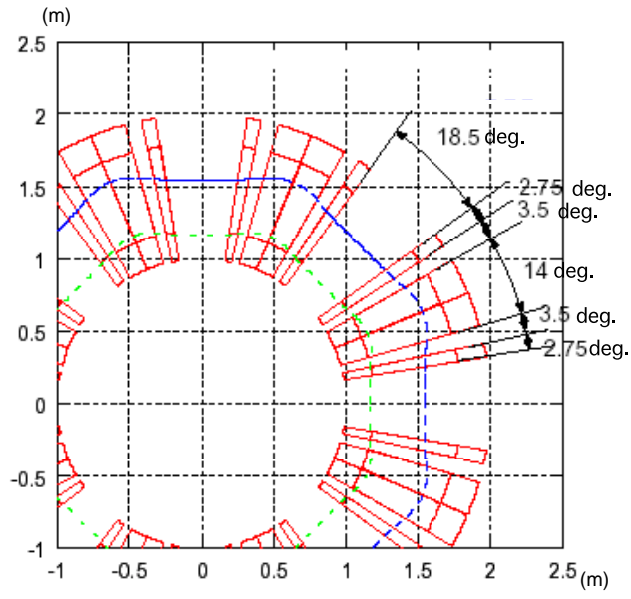

Figure 5: Lattice structure of the booster ring. Green and Blue lines are the beam orbits corresponding to $r_{i n j}$ and $r_{\text {ext }}$, respectively.

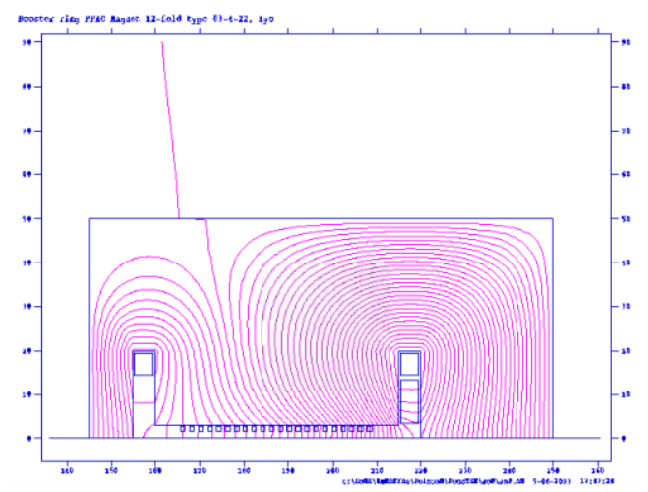

Figure 6: A typical 2D simulation of magnetic field for the magnet in the booster ring.

\section{Main FFAG Synchrotron}

The main accelerator is identical to the $150 \mathrm{MeV}$ FFAG synchrotron which is now tested at KEK, except some mod- 
ifications to achieve the high repetition rate $(100 \mathrm{~Hz})$ in the acceleration in the current FFAG complex. Detailed discussions on magnet design is available in ref. [7]. Since a wideband RF cavity can produce the acceleration voltage $\sim 10 \mathrm{kV}$ at most, we need to have two RF cavities in the ring to complete the acceleration of injected beam bunch from the booster within each repetition period of $\sim 100 \mu \mathrm{s}$. Another diffenence is in the magnets used in the main ring. The cross section of return yoke and the purity of iron are increased to accept a high magnetic flux required for 200 $\mathrm{MeV}$ acceleration, expecting the beam energy upgrade by the reinforcement of power supplies in near future.

\section{Synchronization of Acceleration Timing}

One of the major difficulties in realizing an FFAG complex is to find the exact timing of the beam injection, i.e., when the both of acceleration voltages are in phase. The conventional techniques to find the injection timing cannot be applied in the current FFAG complex because the booster and main rings do not have simple relations on RF frequency or phase unlike the conventional synchrotron complexes. Therefore, we plan to "draw RF waveforms directly" for the booster and main rings so that both RF signals are in-phase at the timing of kick-out from the booster ring. Such RF signals can be easily produced by a signal generator(S.G.) based on DSP, in which the waveform itself can be programmed as much as the memory in the S.G. allows. A typical example of RF signal generated by Tektronix AWG420 is shown in Fig. 7.

\section{CURRENT STATUS AND FUTURE PROSPECTS}

The construction of the building for the FFAG complex named "Innovation Research Laboratory" will be completed at the end of March 2004. This buliding is designed not only for FFAG accelerator complex, but also for the multipurpose usage of the beam from the FFAG complex such as nuclear physics, chemistry, material science and cancer therapy.

The FFAG complex itself will be constructed in the fall of 2004. The first beam from this FFAG complex is expected by the end of March 2005. Basic studies on ADS will be employed just after the beam line between the FFAG complex and KUCA will be ready, expected by the end of March 2006.

\section{REFERENCES}

[1] K. Kawase and M. Inoue, "Neutron Factory Project at KURRI”, APAC 1998, Tsukuba, Japan, p. 104

[2] S. Shiroya, H. Unesaki et al., "Neutronics of Future Neutron Source Based on Accelerator Driven Subcritical Reactor Concept in Kyoto University Research Reactor Institute (KURRI)h, Int. Seminar on Advanced Nucl. Energy Systems toward Zero Release of Radioactive Wastes, 2nd Fujiwara Int. Seminar, Nov. 6-9, 2000, Shizuoka, Japan, Abstracts p. 58.

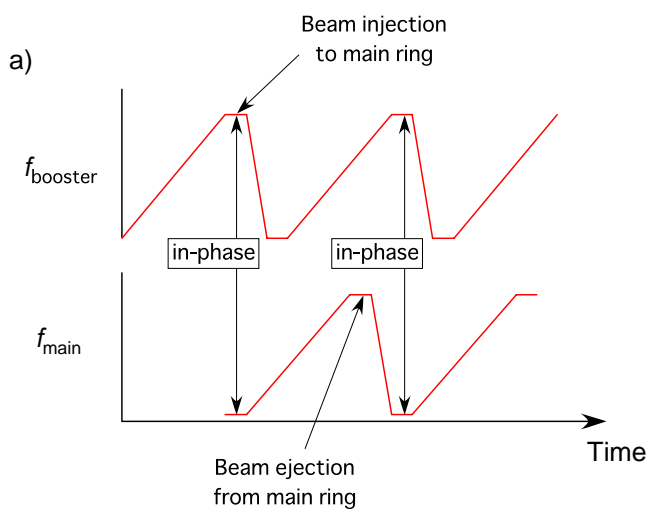

b)

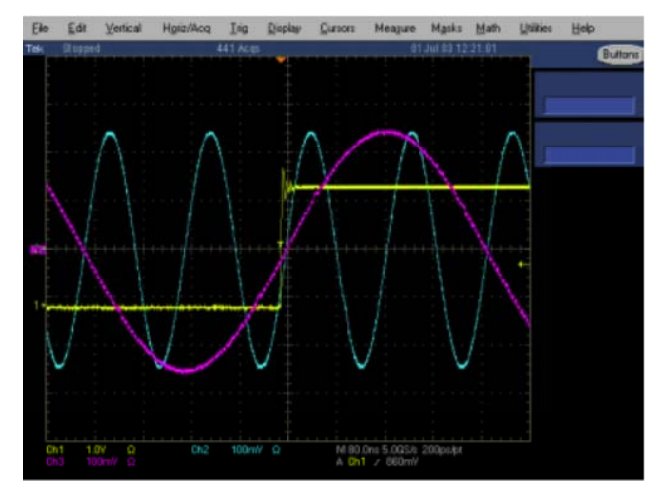

Figure 7: a) A typical RF sweep pattern of the booster and main rings in the current FFAG complex. b) An example of RF outputs from Tektronix AWG420 programmed to follow the RF sweep pattern shown in a). Blue and violet waves are the RF signals and the yellow gate signal shows the desired in-phase timing. In this case, both RF signals are programmed to have the same phase of 0 degree on a desired timing.

[3] S. Shiroya, H. Unesaki et al., Trans. Am. Nucl. Soc., 2001 Annu. Mtg., June 17-21, 2001, Milwaukee, Wisconsin, p. 78.

[4] T.Ohkawa, Proc. of annual meeting of JPS(1953)

[5] Y. Mori et al., "A new type of rf cavity for high intensity proton synchrotron using high permeability magnetic alloy", EPAC 1998, p. 299.

[6] M. Aiba et al., "DEVELOPMENT OF A FFAG PROTON SYNCHROTRON", Proceeding of EPAC 2000, Vienna, Austria, p. 581

[7] T. Adachi et al., "A 150MeV FFAG SYNCHROTRON WITH hRETURN-YOKE FREEh MAGNET”, PAC 2001, Chicago, the United States, p. 3254

[8] M. Aiba et al., "Beam Injection and Extraction in $150 \mathrm{MeV}$ FFAG”, Proceeding of EPAC 2002, Paris, France, p. 1076 